\title{
SECURING OUR CARS
}

2/IIIIIIIIIIIIIIIIIIIIIIIIIIIIIIIIIIIIIIIIIIIIIIIIIIIIIIIIIIIIIIIIIIIIIIIIIIIIIIIIIIIIIIIIIIIIIIIIIIIIIIIIIIIIIIIIIIIIIIIIIIIIIIIIIIIIIIIIIIIIIIIIIIIIIIIIIIIIIIIIIII,

Dear Readers,

By the turn of this decade, BI Intelligence has projected that cars with internet connectivity will comprise $75 \%$ of $92 \mathrm{mn}$ new cars shipped globally. And that from the lows of $13 \%$ in 2015. As for all vehicles on road, McKinsey estimates connected cars could account for $22 \%$, up from $10 \%$ in 2015. Gartner, on the other hand, says by 2020 , there will be a quarter billion connected vehicles on the road, enabling new in-vehicle services and automated driving capabilities.

These are astounding numbers, reason enough for everyone associated with the automotive industry to track developments in the connected car domain, or with autonomous vehicles with utmost interest. That connected cars will form a major element of the Internet of Things (IoT) in the future is a no brainer.

At the same time, nonetheless, cyber security of connected vehicles has emerged as one of the biggest issues facing manufacturers today. Experts expect vulnerabilities will only increase with further growth of connectivity between vehicles, with the infrastructure, with devices or with everything else. And this challenge is not just for the OEMs and suppliers, but everyone else in the value chain - regulators, service providers, insurance agencies, dealers, et al.

In this edition, we have featured a very interesting update on cyber security best practices that have been designed by the Automotive Information Sharing and Analysis Center (Auto-ISAC). These are the first set of best practices that are expected to help combat rising and future threats facing the connected car. The framework designed is expected to provide guidance on how individual companies can enhance automotive cyber security, focusing on seven key areas. Importantly, they refer primarily to US light-duty, on-road vehicles but are applicable to other automotive markets, including heavy-duty and commercial vehicles.

Around the same time, Tel Aviv-based Argus Cyber Security unveiled a multi-layered cyber solution portfolio that includes advanced protection for the entire vehicle ecosystem. Similarly, traditional suppliers, and numerous new organisations are working on solutions to take cyber security challenges posed by the burgeoning connected cars, head-on.

I've personally been quite intrigued by the developments in this area - probably that justifies my second editorial on the subject in four months - and believe it is important for the Indian industry to not just take note of the challenges, but work towards finding answers.

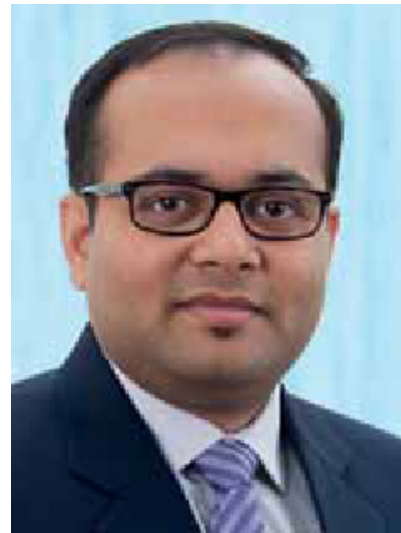

autotechreview.com

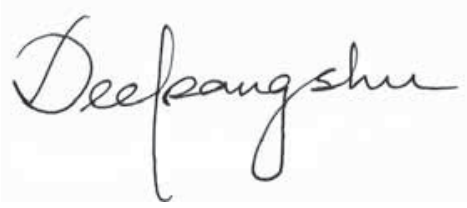

DEEPANGSHU DEV SARMAH

Editor-in-Chief

New Delhi, August 2016 